\title{
An anti-freezing and anti-drying multifunctional gel electrolyte for flexible aqueous zinc-ion batteries
}

\author{
Rui Wang, Minjie Yao, Shuo Huang, Jinlei Tian and Zhiqiang Niu*
}

\begin{abstract}
Aqueous zinc-ion batteries (ZIBs) have attracted immense attention for flexible energy storage devices due to their high safety and low cost. However, conventional flexible aqueous ZIBs will undergo severe capacity loss at subzero temperature due to the inevitably freeze of electrolytes. In addition, under large bending or stretching strains, the encapsulation of devices would be damaged, which causes the evaporation of water in electrolytes and results in device failure. Herein, an anti-freezing and anti-drying gel electrolyte based on polyacrylamide (PAM) and glycerol (Gly) is developed. The strong hydrogen-bonding interactions between PAM or Gly and water molecules not only avoid the crystallization of the gel electrolyte at low temperatures, but also constrain the free water and restrict its evaporation. Therefore, such gel electrolyte displays a high ionic conductivity of $9.65 \times 10^{-5} \mathrm{~S} \mathrm{~cm}^{-1}$ at $-40^{\circ} \mathrm{C}$. Furthermore, it can restrict the dehydration process when the electrolyte is exposed to ambient environment. The flexible ZIBs based on such gel electrolyte exhibit excellent electrochemical performance at $-40^{\circ} \mathrm{C}$ and the devices without encapsulation retain $98 \%$ of their initial capacity in ambient condition after 30 days. This work provides a route to design anti-freezing and anti-drying gel electrolytes for aqueous energy storage devices.
\end{abstract}

Keywords: gel electrolytes, anti-freezing, anti-drying, glycerol, aqueous zinc-ion batteries

\section{INTRODUCTION}

Aqueous zinc-ion batteries (ZIBs) are regarded as a promising candidate for flexible energy storage devices due to their intrinsically high safety and low cost [1-15]. However, conventional flexible aqueous ZIBs are not capable of working at low temperatures, where electrolytes will inevitably freeze and further restrict the ionic transportation, resulting in the degradation of their electrochemical performance [16-20]. In addition, under large bending or stretching strains, the packages of flexible aqueous ZIBs would be damaged, which causes the evaporation of water in electrolytes [21-23]. The loss of water can seriously affect the migration of ions in electrolytes and result in the failure of devices [24-26]. Consequently, it is highly desired to develop anti-freezing and anti-drying electrolytes to meet the demands of flexible aqueous ZIBs.

Gel electrolytes combine the advantages of high ionic conductivity of liquid components and excellent mechanical properties of polymer matrices, which have been the most commonly used electrolytes of flexible energy storage devices [27-30]. Generally, the anti-freezing property of gel electrolytes could be realized by the addition of organic solvents (e.g., ethylene glycol (EG), glycerol (Gly) and dimethyl sulfoxide) or soluble ions (e.g., sulfuric acid, potassium hydroxide and zinc chloride) with high concentrations [31-38]. These solutes with high concentrations could inhibit the crystallization of water and depress the freezing points of electrolytes. However, these gel electrolytes often exhibit limited abilities to restrict free water when they are exposed to ambient environments. The key of designing gel electrolytes with both anti-freezing and anti-drying properties is to weaken the original hydrogen bonds between their water molecules. Polyacrylamide (PAM), which is usually utilized as the polymer matrix of gel electrolytes, often forms strong hydrogen bonds with water molecules due to its abundant amide groups [39-42]. In addition, as a common cryoprotectant and humectant, Gly can provide a large number of hydroxyl groups for constructing strong hydrogen bonds with water molecules [43-46]. Consequently, the combination of the PAM matrix and Gly additive would weaken the hydrogen bonds between water molecules in gel electrolytes, which not only avoids the crystallization of gel electrolytes at low temperatures, but also retards the dehydration of gel electrolytes when they are exposed to ambient conditions. Inspired by this, we developed an antifreezing and anti-drying gel electrolyte based on PAM and Gly. Owing to the strong hydrogen-bonding interactions between PAM or Gly and water molecules, the gel electrolyte displays a low freezing point and excellent drying tolerance. As a result, the gel electrolyte possesses high ionic conductivity at low temperatures and long-term electrochemical stability at ambient environment. Furthermore, the excellent mechanical properties of such hydrogel endow it with ability to serve as the electrolyte of flexible ZIBs. As a proof of concept, flexible aqueous ZIBs were fabricated based on this gel electrolyte. Impressively, they exhibited excellent electrochemical stability under different bending states even at low temperatures. And the devices also displayed high capacity retention rate when they were exposed to external environment.

Key Laboratory of Advanced Energy Materials Chemistry (Ministry of Education), Renewable Energy Conversion and Storage Center, College of Chemistry, Nankai University, Tianjin 300071, China

* Corresponding author (email: zqniu@nankai.edu.cn) 


\section{EXPERIMENTAL SECTION}

Preparation of anti-freezing and anti-drying gel electrolytes Acrylamide $(2 \mathrm{~g})$, ammonium persulphate $(0.25 \mathrm{~g})$, methylenebis-acrylamide $(1.5 \mathrm{mg})$ and zinc trifluoromethanesulfonate $\left(\mathrm{Zn}\left(\mathrm{CF}_{3} \mathrm{SO}_{3}\right)_{2}, 2.8 \mathrm{~g}\right)$ were dispersed in $10 \mathrm{~mL}$ deionized water to obtain a homogeneous solution. Then Gly with various volume percentages to water was added into the mixed solution. The mixtures were deoxygenized and then heated at $90^{\circ} \mathrm{C}$ for $2 \mathrm{~h}$ for polymerization. After being cooled to room temperature, the anti-freezing and anti-drying gel electrolytes were obtained.

\section{Preparation of flexible aqueous ZIBs}

Polyaniline (PANI) nanorods were prepared according to the previous literature [47]. PANI, single-walled carbon nanotubes (SWCNTs) and polyvinylidene fluoride were mixed with a weight ratio of 7:2:1 in $N$-methyl-2-pyrrolidone, forming a slurry, which was then coated on steel meshes. After vacuum drying at $60^{\circ} \mathrm{C}$ for $24 \mathrm{~h}$, the SWCNTs/PANI cathodes were obtained. The SWCNTs/PANI cathodes, filter paper, and $\mathrm{Zn}$ foil anodes were stacked together and then immersed into the solution that contains the precursor of gel electrolytes. All the components were deoxygenized and then heated at $90^{\circ} \mathrm{C}$ for $2 \mathrm{~h}$ for polymerization. After being cooled to room temperature, the flexible aqueous ZIBs were obtained.

\section{Characterizations and electrochemical measurements}

The morphologies of materials, electrodes and freeze-dried gel electrolytes were characterized via a scanning electron microscope (SEM, JEOL JSM7500F and Phenom XL) and transmission electron microscope (TEM, Talos F200X G2). The Fourier transform infrared (FTIR) spectra of gel electrolytes were collected through Bruker Tensor II. The tensile tests of gel electrolytes were measured on a dynamic mechanical analyzer (TA DMA Q800). The thermal properties of gel electrolytes were characterized using a differential scanning calorimeter (DSC, NETZSCH). The samples were first equilibrated at $25^{\circ} \mathrm{C}$ and then cooled at a rate of $5^{\circ} \mathrm{C} \mathrm{min}^{-1}$ to $-70^{\circ} \mathrm{C}$. After an isothermal period of at least $90 \mathrm{~min}$, the samples were heated up at $2^{\circ} \mathrm{C} \mathrm{min}^{-1}$ to the initial equilibration temperature $\left(25^{\circ} \mathrm{C}\right)$. The ionic conductivity of gel electrolytes was calculated by bulk resistance, which was measured on an electrochemical workstation (Zahner, IM6ex) with an amplitude of $5 \mathrm{mV}$ in the frequency ranging from $100 \mathrm{kHz}$ to $1 \mathrm{~Hz}$. The ionic conductivity $\sigma$ was calculated by:

$\sigma=\frac{l}{R A}$,

where $l, R$, and $A$ represent the thickness, the bulk resistance, and the test area of the gel electrolyte, respectively. Cyclic voltammetry (CV) curves of ZIBs were tested on an electrochemical workstation (CHI Instruments, CHI660E). Galvanostatic charge/ discharge (GCD) curves were recorded on a battery test system (LAND CT2001A). Electrochemical impedance spectroscopy curves were measured in the frequency range from $100 \mathrm{kHz}$ to $100 \mathrm{mHz}$ on an electrochemical workstation (Zahner, IM6ex). The specific capacity of ZIBs was calculated based on the mass of the PANI.

\section{Computational methods}

The binding energy calculations were performed using the density functional theory (DFT) program $\mathrm{DMol}^{3}$ in Material
Studio. The physical wave functions were expanded in terms of numerical basis sets, $\mathrm{Dmol}^{3} / \mathrm{GGA}-\mathrm{PBE} / \mathrm{DNP}$ (3.5) basis set. The core electrons were treated with DFT semi-core pseudo potentials. The exchange-correlation energy was calculated with Perdew-Burke-Ernzerhof (PBE) generalized gradient approximation (GGA). A Fermi smearing of $0.005 \mathrm{Ha}(1 \mathrm{Ha}=27.211 \mathrm{eV})$ and a global orbital cutoff of $5.2 \AA$ were employed. The convergence criteria for the geometric optimization and energy calculation were set as follows: (a) a self-consistent field tolerance of $1.0 \times$ $10^{-6} \mathrm{Ha}$ atom $^{-1}$; (b) an energy tolerance of $1.0 \times 10^{-5} \mathrm{Ha} \mathrm{atom}^{-1}$; (c) a maximum force tolerance of $0.002 \mathrm{Ha}^{-1}$; and (d) a maximum displacement tolerance of $0.005 \AA$.

\section{RESULTS AND DISCUSSION}

The gel electrolyte was synthesized via the free radical polymerization by an acrylamide solution of Gly and zinc trifluoromethanesulfonate $\left(\mathrm{Zn}\left(\mathrm{CF}_{3} \mathrm{SO}_{3}\right)_{2}\right)$. Since such polymerization is a process of converting polymer monomer solution into hydrogel, the shapes of resultant electrolytes could be readily designed by injecting the precursor solution into customized molds (Fig. S1), certifying their outstanding processability. During the polymerization process, acrylamide molecules cross-linked with each other through covalent bonds to construct PAM matrix with three-dimensional (3D) porous structure (Fig. 1a). The size of the pores is in a range of $1-3 \mu \mathrm{m}$, as suggested by the SEM image of the freeze-dried gel electrolyte (Fig. 2a). The 3D porous architecture ensures the gel electrolyte with excellent mechanical properties and fast ionic transportation. Furthermore, since Gly and PAM chains possess abundant hydroxyl groups and amide groups, respectively, the original hydrogen bonds between water molecules are destructed, and the stronger hydrogen-bonding interactions are formed between PAM or Gly and water molecules, as suggested by DFT calculations (Fig. 1b and Fig. S2). During the DFT calculation, the binding energy ( $\left.E_{\text {binding }}\right)$ represents the intensity of the interaction between the components in the system and it can be calculated according to this equation:

$E_{\text {binding }}=E_{\text {total }}-\sum E_{\text {component }}$,

where $E_{\text {total }}$ is the total energy of the system and $E_{\text {component }}$ is the energy of each component. The higher absolute value of the negative $E_{\text {binding }}$ represents a stronger interaction [48]. The binding energy of PAM- $\mathrm{H}_{2} \mathrm{O}\left(-37.76 \mathrm{Kcal} \mathrm{mol}^{-1}\right)$ and $\mathrm{Gly}-\mathrm{H}_{2} \mathrm{O}$ $\left(-40.66 \mathrm{Kcal} \mathrm{mol}^{-1}\right)$ is much lower than the case of $\mathrm{H}_{2} \mathrm{O}-\mathrm{H}_{2} \mathrm{O}$ $\left(-5.08 \mathrm{Kcal} \mathrm{mol}^{-1}\right)$. This result proves that PAM- $\mathrm{H}_{2} \mathrm{O}$ and Gly$\mathrm{H}_{2} \mathrm{O}$ systems are more stable than the $\mathrm{H}_{2} \mathrm{O}-\mathrm{H}_{2} \mathrm{O}$ system, suggesting that water molecules prefer to bond with PAM and Gly molecules rather than other water molecules. Furthermore, the binding energy of PAM- $\mathrm{H}_{2} \mathrm{O}-\mathrm{Gly}\left(-64.8 \mathrm{Kcal} \mathrm{mol}^{-1}\right)$ is also much lower than the case of $\mathrm{H}_{2} \mathrm{O}-\mathrm{H}_{2} \mathrm{O}$, indicating that the PAM$\mathrm{H}_{2} \mathrm{O}$-Gly system possesses higher stability and stronger hydrogen-bonding interactions. Meanwhile, the PAM- $\mathrm{H}_{2} \mathrm{O}-\mathrm{Gly}$ system is more stable than the common gel electrolyte systems such as PAM- $\mathrm{H}_{2} \mathrm{O}-\mathrm{EG}\left(-53.1 \mathrm{Kcal} \mathrm{mol}^{-1}\right)$, poly(vinyl alcohol) (PVA)- $\mathrm{H}_{2} \mathrm{O}$-Gly $\left(-52.6 \mathrm{Kcal} \mathrm{mol}^{-1}\right)$, and poly(acrylic acid) (PAA)- $\mathrm{H}_{2} \mathrm{O}-\mathrm{Gly}\left(-60.4 \mathrm{Kcal} \mathrm{mol}^{-1}\right.$ ) (Fig. 1c). This result reveals that sufficient hydroxyl groups and amide groups are beneficial to constructing strong hydrogen-bonding interactions with water molecules. Additionally, the strong hydrogen-bonding interactions of these systems can be observed more intuitively through the average hydrogen bond length [49-51]. As shown in 


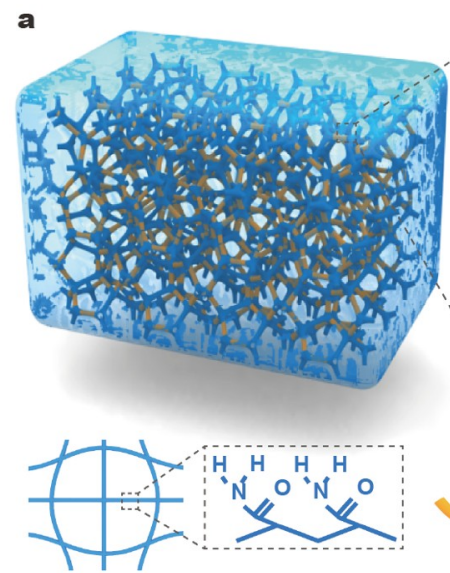

PAM chain
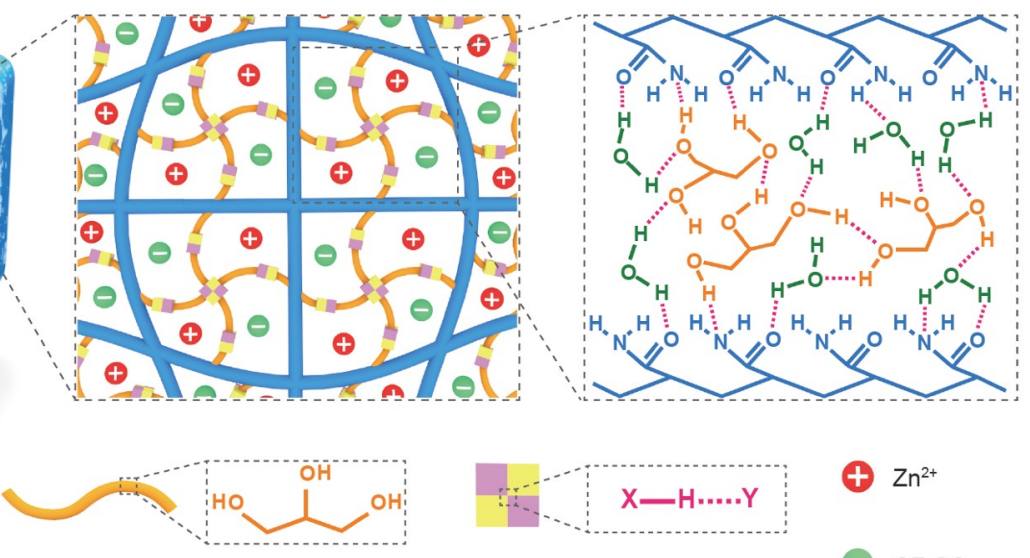

Glycerol

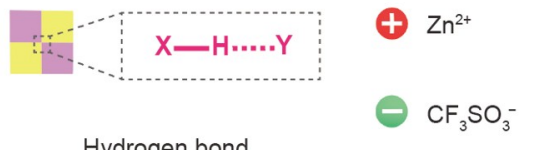

Hydrogen bond

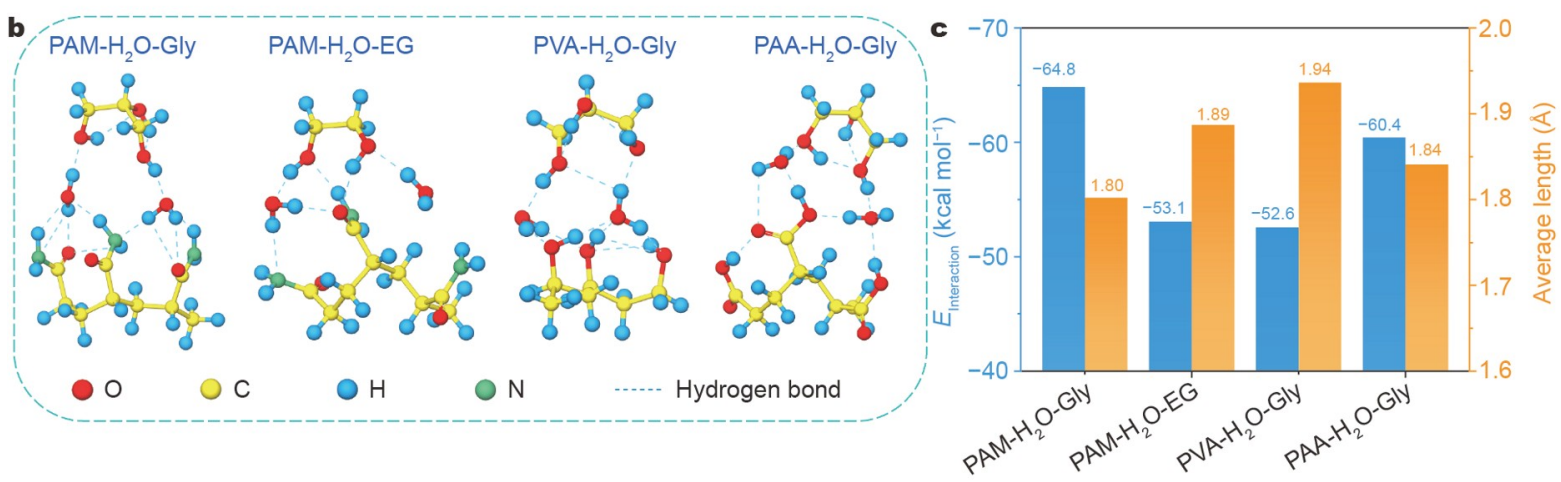

Figure 1 (a) Configuration and bonding mechanism of the anti-freezing and anti-drying gel electrolyte. (b) DFT optimized structures, (c) calculated interaction energies and average lengths of hydrogen bonds of PAM- $\mathrm{H}_{2} \mathrm{O}-\mathrm{Gly}, \mathrm{PAM}-\mathrm{H}_{2} \mathrm{O}-\mathrm{EG}, \mathrm{PVA}-\mathrm{H}_{2} \mathrm{O}-\mathrm{Gly}$, and PAA- $\mathrm{H}_{2} \mathrm{O}-\mathrm{Gly}$.
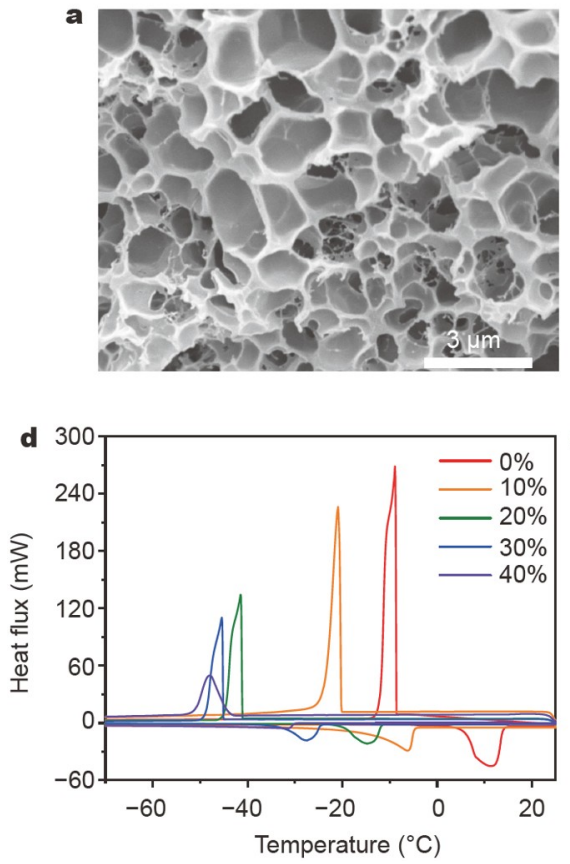
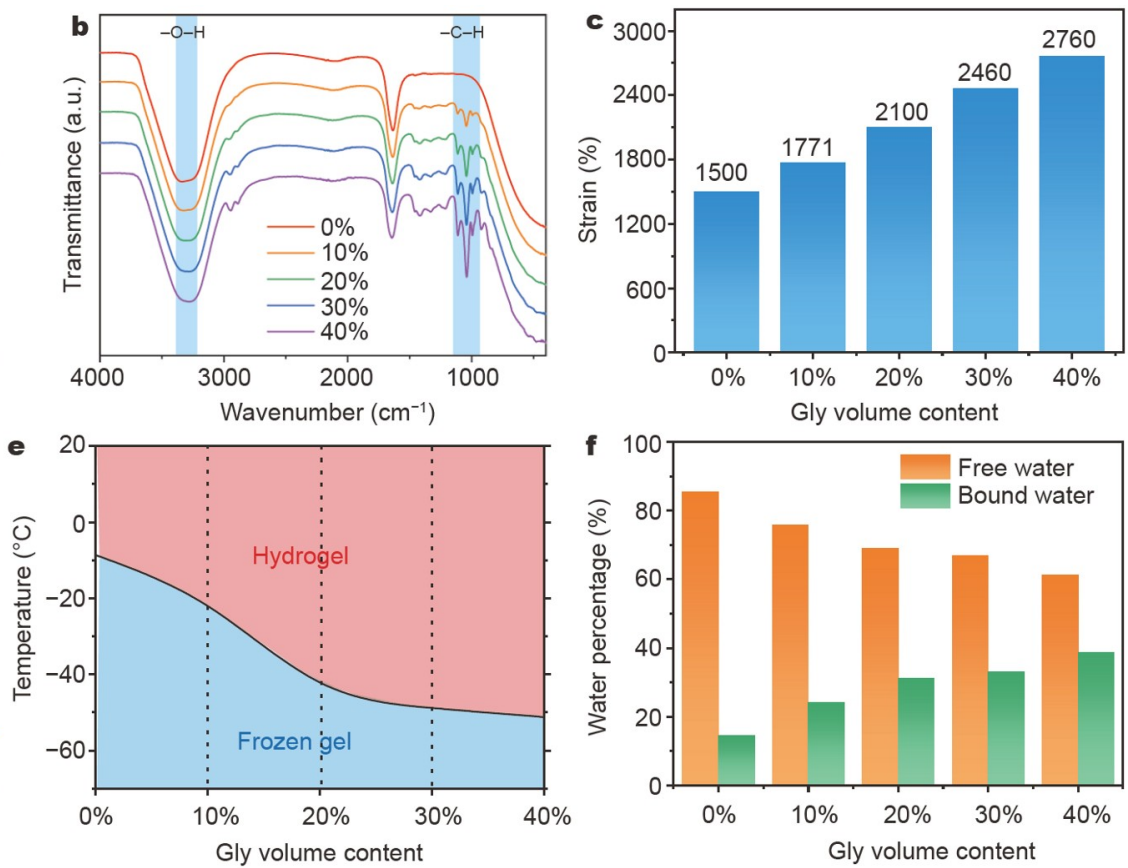

Figure 2 (a) SEM image of PAM- $\mathrm{H}_{2} \mathrm{O}-\mathrm{Gly}$. (b) FTIR spectra, (c) tensile strain, (d) DSC curves, (e) phase diagram and (f) contents of free water and bound water of PAM- $\mathrm{H}_{2} \mathrm{O}-\mathrm{Gly}$ with different contents of Gly. 
Fig. 1c, the average hydrogen bond length of $\mathrm{PAM}-\mathrm{H}_{2} \mathrm{O}-\mathrm{Gly}$ is shorter in comparison with the cases of common gel electrolyte systems, suggesting a higher stability of the PAM- $\mathrm{H}_{2} \mathrm{O}-\mathrm{Gly}$ system, which is consistent with the results of binding energy. Furthermore, the hydrogen bonds were further identified by their characteristic peaks in the FTIR spectra. As shown in Fig. $2 b$, the characteristic peak at $3270 \mathrm{~cm}^{-1}$ is ascribed to the hydroxyl $[52,53]$. With the increase of the Gly volume content from $0 \%$ to $40 \%$, this characteristic peak gradually shifts to the lower wavenumber in the $\mathrm{PAM}-\mathrm{H}_{2} \mathrm{O}$-Gly system, indicating an enhancement of intermolecular hydrogen-bonding interactions due to the formation of more hydrogen bonds between Gly and $\mathrm{H}_{2} \mathrm{O}$ molecules.

The hydrogen bonds also play an important role in the mechanical properties of gel electrolytes [54]. After introducing Gly into the PAM matrix, more hydrogen bonds will form between Gly molecules and PAM polymer chains, which can dissipate the energy between PAM chains under deformation and homogenize the polymer network [55]. As a result, the combination of covalent cross-linking bonding and physical hydrogen bonding endows gel electrolytes with excellent flexibility and enhanced strain tolerance [56-58]. As shown in Fig. S3, the strain tolerance of the PAM- $\mathrm{H}_{2} \mathrm{O}$-Gly electrolyte will be remarkably improved with addition of Gly. When the Gly volume content reaches $40 \%$, the electrolyte displays a high strain tolerance of $2760 \%$ (Fig. $2 c$ and Fig. S4), which is much larger than the case of the electrolyte without Gly. Apart from the enhanced strain tolerance of the gel electrolyte, the freezing point of the gel electrolyte will be significantly reduced by the addition of Gly since the strong hydrogen-bonding interactions between Gly and water can disrupt the formation of ice crystal in electrolytes at low temperatures. The decreased freezing points of gel electrolytes were detected by DSC measurement. As shown in Fig. 2d, the exothermic peak in DSC curves represents the crystallization of the gel electrolyte, where the liquid phase in the gel electrolyte will transform into solid phase [59]. When the Gly volume content increases from $0 \%$ to $40 \%$, the exothermic peak of gel electrolytes gradually shifts towards left, indicating the decrease of their freezing points [60-62]. When the Gly volume content reaches $40 \%$, the gel electrolyte exhibits an ultralow freezing point of $-51^{\circ} \mathrm{C}$, which is much lower than the case $\left(-9^{\circ} \mathrm{C}\right)$ of the gel electrolyte without addition of Gly (Fig. 2e). Furthermore, the low freezing point of gel electrolytes can be deeply understood by the content of free water in gel electrolytes. Free water has almost no interaction with the polymer network. Therefore, free water displays similar physical properties with pure water and it will unavoidably freeze at low temperatures, which causes the crystallization of gel electrolytes [51]. The content of free water $\left(W_{\text {free }}\right)$ can be calculated through the enthalpy change $(\Delta H)$ during the heating process in the DSC curve by this equation:

$W_{\text {free }}=\frac{\Delta H}{\Delta H_{0}} \times 100 \%$,

where $\Delta H_{0}$ is the enthalpy value of pure water $\left(334 \mathrm{~J} \mathrm{~g}^{-1}\right)$. As shown in Fig. 2f, with the increase of the Gly content, the content of free water in gel electrolytes is gradually decreased, leading to the low freezing point of gel electrolytes. To demonstrate their freezing tolerance more intuitively, the states of gel electrolytes with different Gly contents under different environmental temperatures were further monitored. It can be seen that when the addition of Gly is up to $20 \mathrm{vol} \%$, the PAM-
$\mathrm{H}_{2} \mathrm{O}$-Gly electrolyte would not freeze even at $-40^{\circ} \mathrm{C}$ (Fig. 3a and Fig. S5), displaying an outstanding anti-freezing behavior. The ionic conductivity at low temperatures is a key feature to evaluate anti-freezing electrolytes. At $-40^{\circ} \mathrm{C}, \mathrm{PAM}-\mathrm{H}_{2} \mathrm{O}-\mathrm{Gly}-20 \%$ still retains an excellent ionic conductivity of $9.65 \times 10^{-5} \mathrm{~S} \mathrm{~cm}^{-1}$ due to its low freezing point (Fig. $3 \mathrm{~b}$ and Fig. S6), though the ionic conductivity of the $\mathrm{PAM}-\mathrm{H}_{2} \mathrm{O}-\mathrm{Gly}$ electrolyte is gradually reduced with the increase of Gly content at room temperature, which is ascribed to the decreased content of free water in the electrolyte [63].

The excellent mechanical properties and ionic conductivity endow PAM- $\mathrm{H}_{2} \mathrm{O}-\mathrm{Gly}$ hydrogel with the ability to serve as the electrolyte of flexible ZIBs. As a proof of concept, flexible aqueous ZIBs were fabricated based on the PAM- $\mathrm{H}_{2} \mathrm{O}$-Gly electrolyte, where SWCNTs/PANI served as cathodes and $\mathrm{Zn}$ foil acted as anodes (Fig. $3 \mathrm{c}$ and Fig. S7). The ZIBs based on the PAM$\mathrm{H}_{2} \mathrm{O}-\mathrm{Gly}-20 \%$ electrolyte deliver a discharge capacity of $67.8 \mathrm{~mA} \mathrm{~h} \mathrm{~g}^{-1}$ under room temperature at $1 \mathrm{~A} \mathrm{~g}^{-1}$ (Fig. 3d, Figs S8 and S9a), which is comparable to those of previously reported PANI-based ZIBs. Impressively, owing to the excellent anti-freezing behavior and high ionic conductivity of the PAM$\mathrm{H}_{2} \mathrm{O}-\mathrm{Gly}-20 \%$ electrolyte, the ZIBs still exhibit a specific capacity of $52 \mathrm{~mA} \mathrm{~h} \mathrm{~g}^{-1}$ at $-40^{\circ} \mathrm{C}$. In contrast, the capacity of ZIBs based on the PAM- $\mathrm{H}_{2} \mathrm{O}-\mathrm{Gly}-0$ electrolyte deteriorates seriously with the decrease of temperature, which ultimately causes device failure at $-40^{\circ} \mathrm{C}$. The severe performance degradation possibly results from the faded ionic conductivity of the gel electrolyte at low temperatures. Furthermore, even at different current densities, the ZIBs based on the $\mathrm{PAM}-\mathrm{H}_{2} \mathrm{O}-\mathrm{Gly}-20 \%$ electrolyte also display outstanding discharge capacity at $-40^{\circ} \mathrm{C}$ (Fig. S9b). Moreover, even after charge/discharge for 500 cycles, the ZIBs based on PAM- $\mathrm{H}_{2} \mathrm{O}-\mathrm{Gly}-20 \%$ still maintain $76.8 \%$ of its initial capacity at $-40^{\circ} \mathrm{C}$, exhibiting a superior cycling performance at low temperatures (Fig. S9c). To demonstrate the excellent electrochemical performance of anti-freezing ZIBs at low temperatures more visually, two ZIBs based on PAM- $\mathrm{H}_{2} \mathrm{O}-\mathrm{Gly}-20 \%$ were integrated in series to power a thermometer at $-40^{\circ} \mathrm{C}$ (Fig. 3e). It is noted that the thermometer works well under low temperatures. More impressively, at cold condition, the flexible antifreezing ZIBs also exhibit stable electrochemical performance under different bending states due to their all-in-one configurations and excellent freezing tolerance of electrolytes. As shown in Fig. 3f, when the ZIBs were bent into cylinders and then returned to flat state during the cycling process at $-40^{\circ} \mathrm{C}$, their capacities were almost unchanged in comparison with the case at the initial state, indicating that the flexible anti-freezing ZIBs possess great promise for the practical application in cold conditions.

In addition to anti-freezing behavior, the PAM- $\mathrm{H}_{2} \mathrm{O}-\mathrm{Gly}$ electrolyte also exhibits a remarkable anti-drying performance in ambient environment since the evaporation of water in electrolytes is inhibited. As shown in Fig. 4a, b, dehydration tests of PAM- $\mathrm{H}_{2} \mathrm{O}-\mathrm{Gly}$ electrolytes with different contents of Gly were carried out at $25^{\circ} \mathrm{C}$ and $70 \%$ humidity for 30 days, where the volume change and weight retention of electrolytes were recorded. The results reveal that the electrolyte with higher content of Gly exhibits superior anti-dehydration performance. When the addition of Gly reaches $40 \mathrm{vol} \%$, there is only $6 \%$ weight loss of the gel electrolyte after 30 days, whereas the gel electrolyte without addition of Gly loses $41 \%$ of its weight after 10 days and finally converts into hard scaffolds without water. The out- 

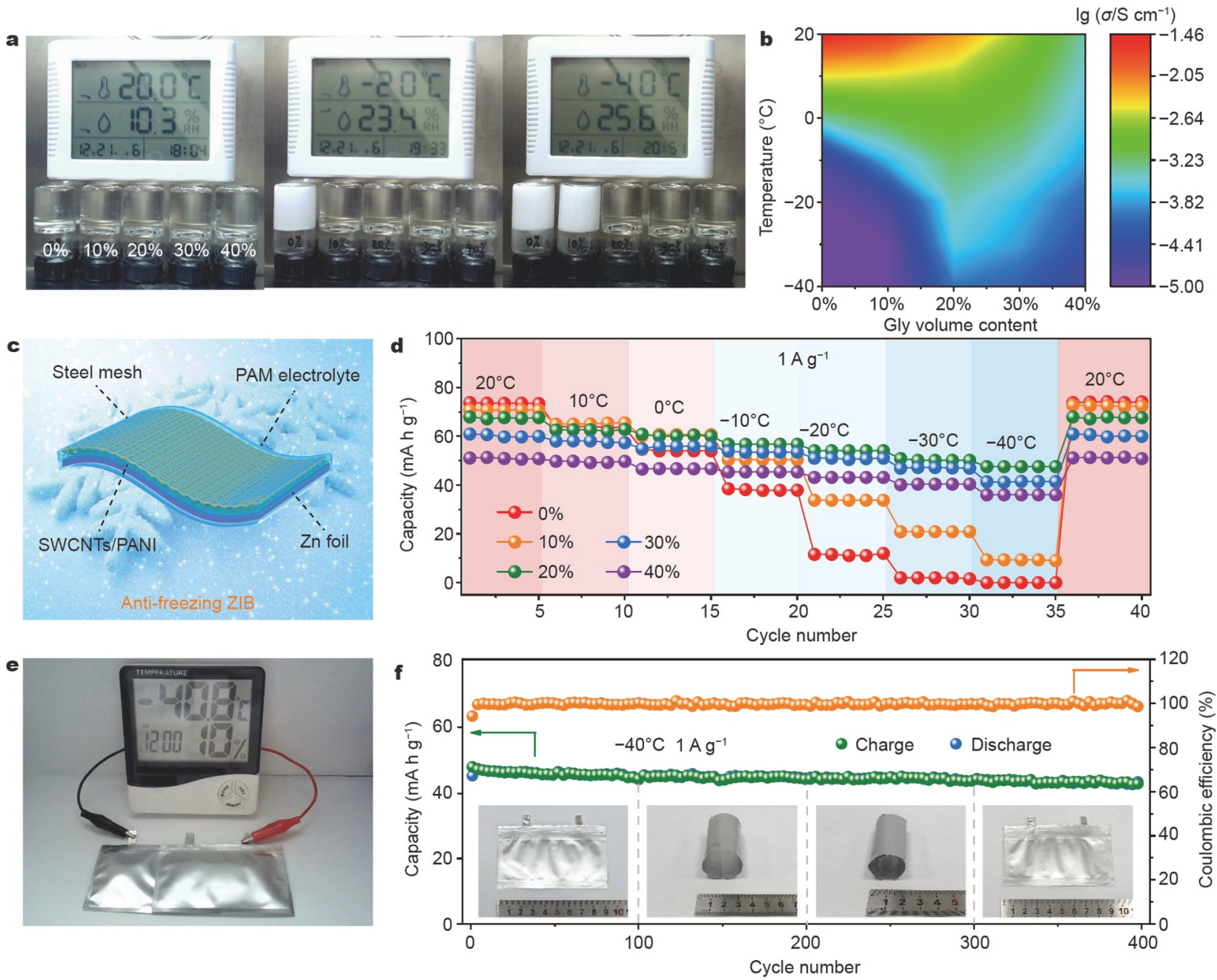

Figure 3 (a) Optical images and (b) ionic conductivities of PAM- $\mathrm{H}_{2} \mathrm{O}-\mathrm{Gly}$ with different contents of Gly from 20 to $-40^{\circ} \mathrm{C}$. (c) The configuration of flexible anti-freezing ZIBs. (d) Capacities of flexible anti-freezing ZIBs at different temperatures. (e) Optical image of a thermometer powered by two flexible antifreezing ZIBs in series at $-40^{\circ} \mathrm{C}$. (f) Cycling performance of flexible anti-freezing ZIBs at different bending states under $-40^{\circ} \mathrm{C}$ at $1 \mathrm{~A} \mathrm{~g}^{-1}$. The insets are the optical images of flexible anti-freezing ZIBs at different bending states.

standing anti-drying performance of gel electrolytes is attributed to the strong hydrogen-bonding interactions between Gly and water molecules, which effectively suppress the evaporation of free water in electrolytes [64-66]. Furthermore, such excellent anti-dehydration behavior endows gel electrolytes with high ionic conductivity even after long-term exposure to ambient environment. As shown in Fig. 4c, the PAM- $\mathrm{H}_{2} \mathrm{O}-\mathrm{Gly}-20 \%$ electrolyte retains a superior ionic conductivity of $2.03 \times$ $10^{-4} \mathrm{~S} \mathrm{~cm}^{-1}$ after 30 days. However, when the Gly volume content of gel electrolytes is further increased, the ionic conductivities of electrolytes gradually decrease, probably because excessive Gly restricts most of the free water and hinders the migration of ions in electrolytes [47,67-69]. The excellent antidehydration performance of the $\mathrm{PAM}-\mathrm{H}_{2} \mathrm{O}-\mathrm{Gly}$ electrolyte makes it promising to serve as the electrolyte of flexible antidrying ZIBs. The flexible ZIBs based on the PAM- $\mathrm{H}_{2} \mathrm{O}-\mathrm{Gly}$ electrolyte were assembled without encapsulation. The charge/ discharge performance of such devices was tested at $25^{\circ} \mathrm{C}$ and $70 \%$ humidity. After 30 days, the capacity of ZIBs based on the PAM- $\mathrm{H}_{2} \mathrm{O}-\mathrm{Gly}-20 \%$ electrolyte still retains $66.4 \mathrm{~mA} \mathrm{~h} \mathrm{~g}^{-1}$, exhibiting a remarkable capacity retention of $98 \%$ (Fig. $4 \mathrm{~d}$ and
Fig. S10). In contrast, the capacity of ZIBs based on the PAM$\mathrm{H}_{2} \mathrm{O}-\mathrm{Gly}-0$ electrolyte fades rapidly, resulting in device failure only after 2 days. Consequently, the outstanding anti-dehydration behavior of electrolytes greatly enhances the durability of devices.

\section{CONCLUSIONS}

In summary, the PAM- $\mathrm{H}_{2} \mathrm{O}$-Gly gel electrolytes with antifreezing and anti-drying behaviors were developed through constructing a strong hydrogen-bonding system. Since the hydrogen-bonding interactions between PAM or Gly and water molecules were stronger than the cases of water, the formation of crystal lattices in electrolytes at low temperatures was disrupted and the evaporation of free water was also suppressed. Therefore, such gel electrolytes displayed high ionic conductivity at $-40^{\circ} \mathrm{C}$ and restricted their dehydration process in ambient environment. Based on this anti-freezing and anti-drying gel electrolyte, the flexible ZIBs were fabricated. They exhibited excellent electrochemical performance at $-40^{\circ} \mathrm{C}$ and retained $98 \%$ of their initial capacity without encapsulation after 30 days. This work broadens the horizons of designing anti-freezing and 
a
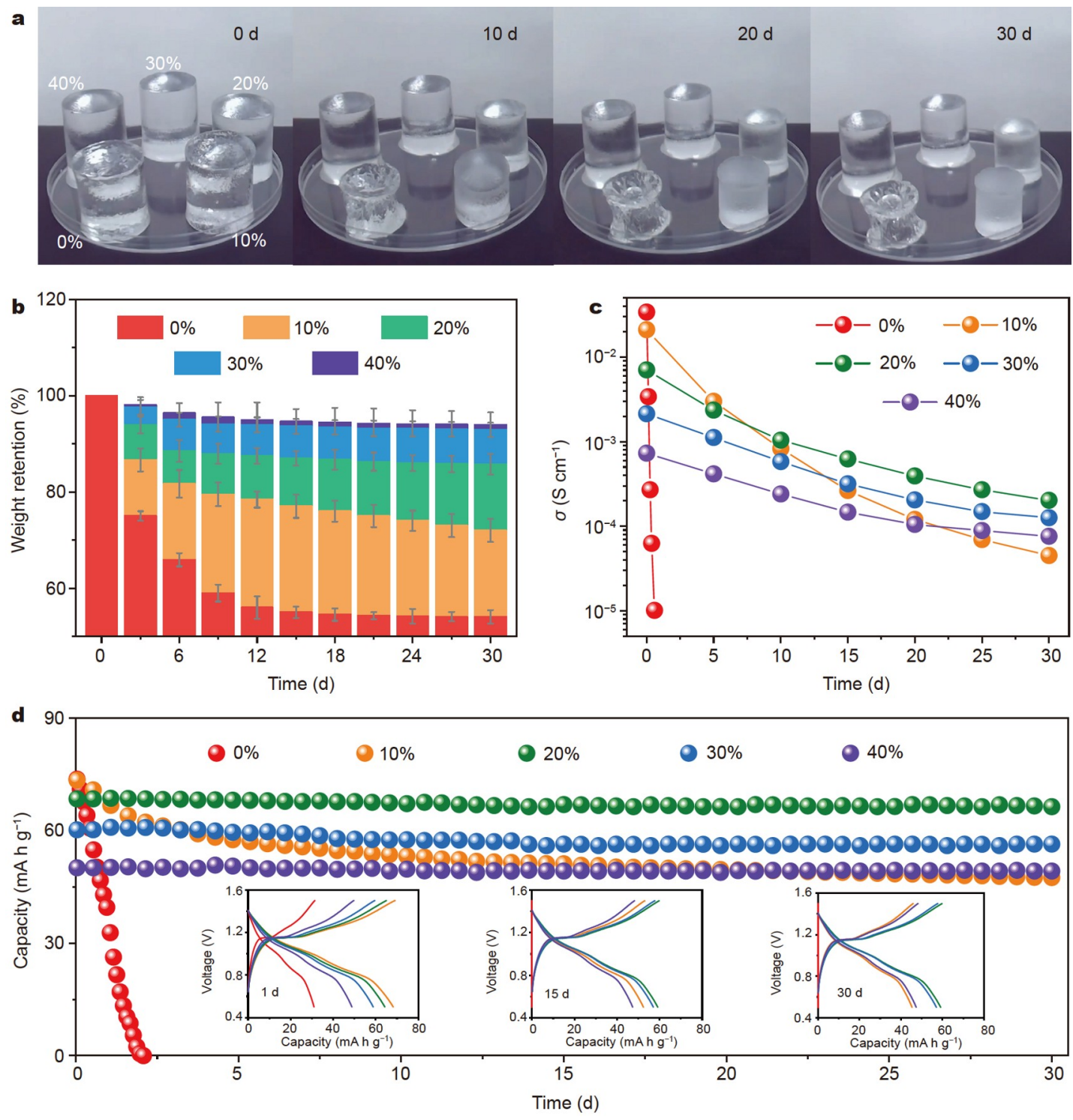

Figure 4 (a) Optical images, (b) weight retentions and (c) ionic conductivities of PAM- $\mathrm{H}_{2} \mathrm{O}-\mathrm{Gly}$ with different Gly contents after 30 days in ambient environment. (d) Cycling performance of flexible anti-drying ZIBs with different contents of Gly. The insets are GCD curves of flexible anti-drying ZIBs after 1,15 , and 30 days, respectively.

anti-drying gel electrolytes for flexible energy storage devices.

Received 27 October 2021; accepted 6 December 2021; published online 12 January 2022

1 Zhong C, Deng Y, Hu W, et al. A review of electrolyte materials and compositions for electrochemical supercapacitors. Chem Soc Rev, 2015, 44: 7484-7539

2 Huang S, Zhu J, Tian J, et al. Recent progress in the electrolytes of aqueous zinc-ion batteries. Chem Eur J, 2019, 25: 14480-14494

3 Wang Z, Li H, Tang Z, et al. Hydrogel electrolytes for flexible aqueous energy storage devices. Adv Funct Mater, 2018, 28: 1804560

4 Cheng XB, Zhang R, Zhao CZ, et al. A review of solid electrolyte interphases on lithium metal anode. Adv Sci, 2016, 3: 1500213

5 Xiao Y, Wang $\mathrm{Y}$, Bo $\mathrm{SH}$, et al. Understanding interface stability in solid-state batteries. Nat Rev Mater, 2019, 5: 105-126

6 Chuai M, Yang J, Wang M, et al. High-performance Zn battery with transition metal ions co-regulated electrolytic $\mathrm{MnO}_{2}$. eScience, 2021, doi: 10.1016/j.esci.2021.11.002

7 Wan F, Wang $\mathrm{X}, \mathrm{Bi}$ S, et al. Freestanding reduced graphene oxide/ sodium vanadate composite films for flexible aqueous zinc-ion batteries. Sci China Chem, 2019, 62: 609-615

8 Huang J, Zhou J, Liang S. Guest pre-intercalation strategy to boost the electrochemical performance of aqueous zinc-ion battery cathodes. Acta Phys-Chim Sin, 2021, 37: 2005020

9 Li P, Kim H, Ming J, et al. Quasi-compensatory effect in emerging anode-free lithium batteries. eScience, 2021, 1: 3-12

10 Su L, Liu L, Wang Y, et al. Synergetic ternary metal oxide nanodotsgraphene cathode for high performance zinc energy storage. Chin Chem Lett, 2020, 31: 2358-2364

11 Zhao M, Li XY, Chen X, et al. Promoting the sulfur redox kinetics by mixed organodiselenides in high-energy-density lithium-sulfur batteries. eScience, 2021, 1: 44-52

12 Zhao Y, Zhu Y, Zhang X. Challenges and perspectives for manganesebased oxides for advanced aqueous zinc-ion batteries. InfoMat, 2020, 2: 237-260

13 Zhang M, Liang R, Or T, et al. Recent progress on high-performance cathode materials for zinc-ion batteries. Small Struct, 2021, 2: 2000064

14 Fan X, Liu B, Liu J, et al. Battery technologies for grid-level large-scale electrical energy storage. Trans Tianjin Univ, 2020, 26: 92-103 
15 Zhu YF, Xiao Y, Dou SX, et al. Spinel/post-spinel engineering on layered oxide cathodes for sodium-ion batteries. eScience, 2021, 1: 1327

16 Ngai KS, Ramesh S, Ramesh K, et al. A review of polymer electrolytes: Fundamental, approaches and applications. Ionics, 2016, 22: 1259-1279

17 Liu Z, Liang G, Zhan Y, et al. A soft yet device-level dynamically supertough supercapacitor enabled by an energy-dissipative dual-crosslinked hydrogel electrolyte. Nano Energy, 2019, 58: 732-742

18 Niu Z, Zhou W, Chen X, et al. Highly compressible and all-solid-state supercapacitors based on nanostructured composite sponge. Adv Mater, 2015, 27: 6002-6008

19 Li H, Lv T, Sun H, et al. Ultrastretchable and superior healable supercapacitors based on a double cross-linked hydrogel electrolyte. Nat Commun, 2019, 10: 536

20 Dong C, Xu F, Chen L, et al. Design strategies for high-voltage aqueous batteries. Small Struct, 2021, 2: 2100001

21 Wei J, Wei G, Shang Y, et al. Dissolution-crystallization transition within a polymer hydrogel for a processable ultratough electrolyte. Adv Mater, 2019, 31: 1900248

22 Zhou D, Chen F, Handschuh-Wang S, et al. Biomimetic extremetemperature- and environment-adaptable hydrogels. ChemPhysChem, 2019, 20: 2139-2154

23 Wang Z, Cheng J, Zhou J, et al. All-climate aqueous fiber-shaped supercapacitors with record areal energy density and high safety. Nano Energy, 2018, 50: 106-117

24 Huang S, Wan F, Bi S, et al. A self-healing integrated all-in-one zinc-ion battery. Angew Chem Int Ed, 2019, 58: 4313-4317

25 Huang $\mathrm{Y}$, Zhu M, Huang Y, et al. Multifunctional energy storage and conversion devices. Adv Mater, 2016, 28: 8344-8364

26 Wan F, Zhu J, Huang S, et al. High-voltage electrolytes for aqueous energy storage devices. Batteries Supercaps, 2020, 3: 323-330

27 Liu J, Xie C, Kretzschmann A, et al. Metallopolymer organohydrogels with photo-controlled coordination crosslinks work properly below $0^{\circ} \mathrm{C}$. Adv Mater, 2020, 32: 1908324

$28 \mathrm{Ju} \mathrm{M}, \mathrm{Wu} \mathrm{B}$, Sun S, et al. Redox-active iron-citrate complex regulated robust coating-free hydrogel microfiber net with high environmental tolerance and sensitivity. Adv Funct Mater, 2020, 30: 1910387

29 Chen M, Zhou W, Wang A, et al. Anti-freezing flexible aqueous $\mathrm{Zn}$ $\mathrm{MnO}_{2}$ batteries working at $-35^{\circ} \mathrm{C}$ enabled by a borax-crosslinked polyvinyl alcohol/glycerol gel electrolyte. J Mater Chem A, 2020, 8: 6828-6841

30 Li H, Zhang H, Diemant T, et al. Reversible copper sulfide conversion in nonflammable trimethyl phosphate electrolytes for safe sodium-ion batteries. Small Struct, 2021, 2: 2100035

31 Peng S, Jiang X, Xiang X, et al. High-performance and flexible solidstate supercapacitors based on high toughness and thermoplastic poly(vinyl alcohol)/NaCl/glycerol supramolecular gel polymer electrolyte. Electrochim Acta, 2019, 324: 134874

32 Chen F, Zhou D, Wang J, et al. Rational fabrication of anti-freezing, non-drying tough organohydrogels by one-pot solvent displacement. Angew Chem Int Ed, 2018, 57: 6568-6571

33 Ji X. A perspective of $\mathrm{ZnCl}_{2}$ electrolytes: The physical and electrochemical properties. eScience, 2021, doi: 10.1016/j.esci.2021.10.004

34 Hou J, Yang M, Wang D, et al. Fundamentals and challenges of lithium ion batteries at temperatures between -40 and $60^{\circ} \mathrm{C}$. Adv Energy Mater, 2020, 10: 1904152

35 Jin $\mathrm{X}$, Song $\mathrm{L}$, Yang $\mathrm{H}$, et al. Stretchable supercapacitor at $-30^{\circ} \mathrm{C}$. Energy Environ Sci, 2021, 14: 3075-3085

36 Peng M, Wang L, Li L, et al. Molecular crowding agents engineered to make bioinspired electrolytes for high-voltage aqueous supercapacitors. eScience, 2021, 1: 83-90

37 Liu L, Dou Q, Sun Y, et al. A moisture absorbing gel electrolyte enables aqueous and flexible supercapacitors operating at high temperatures. J Mater Chem A, 2019, 7: 20398-20404

38 Sun Y, Ma H, Zhang X, et al. Salty ice electrolyte with superior ionic conductivity towards low-temperature aqueous zinc ion hybrid capacitors. Adv Funct Mater, 2021, 31: 2101277

39 Mo F, Liang G, Meng Q, et al. A flexible rechargeable aqueous zinc manganese-dioxide battery working at $-20^{\circ} \mathrm{C}$. Energy Environ Sci,
2019, 12: 706-715

40 Ma L, Zhao Y, Ji X, et al. A usage scenario independent "air chargeable" flexible zinc ion energy storage device. Adv Energy Mater, 2019, 9: 1900509

41 Ma L, Chen S, Wang D, et al. Super-stretchable zinc-air batteries based on an alkaline-tolerant dual-network hydrogel electrolyte. Adv Energy Mater, 2019, 9: 1803046

42 Lou Z, Shen G. Flexible image sensors with semiconducting nanowires for biomimic visual applications. Small Struct, 2021, 2: 2000152

43 Han L, Liu K, Wang M, et al. Mussel-inspired adhesive and conductive hydrogel with long-lasting moisture and extreme temperature tolerance. Adv Funct Mater, 2018, 28: 1704195

44 Liu T, Liu M, Dou S, et al. Triboelectric-nanogenerator-based soft energy-harvesting skin enabled by toughly bonded elastomer/hydrogel hybrids. ACS Nano, 2018, 12: 2818-2826

45 Yuk H, Zhang T, Parada GA, et al. Skin-inspired hydrogel-elastomer hybrids with robust interfaces and functional microstructures. Nat Commun, 2016, 7: 12028

46 Yang J, Gao L, Liu M, et al. Advanced biotechnology for cell cryopreservation. Trans Tianjin Univ, 2020, 26: 409-423

47 Wang R, Yao M, Huang S, et al. Sustainable dough-based gel electrolytes for aqueous energy storage devices. Adv Funct Mater, 2021, 31: 2009209

48 Liu A, Kovacik P, Peard N, et al. Monolithic flexible supercapacitors integrated into single sheets of paper and membrane via vapor printing. Adv Mater, 2017, 29: 1606091

49 Li X, Liu L, Wang X, et al. Flexible and self-healing aqueous supercapacitors for low temperature applications: Polyampholyte gel electrolytes with biochar electrodes. Sci Rep, 2017, 7: 1685

50 Tao F, Qin L, Wang Z, et al. Self-healable and cold-resistant supercapacitor based on a multifunctional hydrogel electrolyte. ACS Appl Mater Interfaces, 2017, 9: 15541-15548

51 Jian Y, Handschuh-Wang S, Zhang J, et al. Biomimetic anti-freezing polymeric hydrogels: Keeping soft-wet materials active in cold environments. Mater Horiz, 2020, 8: 351-369

52 Liu X, Taiwo OO, Yin C, et al. Aligned ionogel electrolytes for hightemperature supercapacitors. Adv Sci, 2019, 6: 1801337

53 Evanko B, Boettcher SW, Yoo SJ, et al. Redox-enhanced electrochemical capacitors: Status, opportunity, and best practices for performance evaluation. ACS Energy Lett, 2017, 2: 2581-2590

54 Guo Y, Bae J, Fang Z, et al. Hydrogels and hydrogel-derived materials for energy and water sustainability. Chem Rev, 2020, 120: 7642-7707

55 Vieira MGA, da Silva MA, dos Santos LO, et al. Natural-based plasticizers and biopolymer films: A review. Eur Polym J, 2011, 47: 254-263

56 Zhang Z, Xiao F, Xiao J, et al. Functionalized carbonaceous fibers for high performance flexible all-solid-state asymmetric supercapacitors. J Mater Chem A, 2015, 3: 11817-11823

57 Liu L, Niu Z, Chen J. Unconventional supercapacitors from nanocarbon-based electrode materials to device configurations. Chem Soc Rev, 2016, 45: 4340-4363

58 Liu W, Yan X, Chen J, et al. Novel and high-performance asymmetric micro-supercapacitors based on graphene quantum dots and polyaniline nanofibers. Nanoscale, 2013, 5: 6053-6062

59 Li X, Lou D, Wang $\mathrm{H}$, et al. Flexible supercapacitor based on organohydrogel electrolyte with long-term anti-freezing and anti-drying property. Adv Funct Mater, 2020, 30: 2007291

60 Huang L, Li C, Shi G. High-performance and flexible electrochemical capacitors based on graphene/polymer composite films. J Mater Chem A, 2014, 2: 968-974

61 Zhou Q, Li Y, Huang L, et al. Three-dimensional porous graphene/ polyaniline composites for high-rate electrochemical capacitors. J Mater Chem A, 2014, 2: 17489-17494

62 Kurra N, Jiang Q, Nayak P, et al. Laser-derived graphene: A threedimensional printed graphene electrode and its emerging applications. Nano Today, 2019, 24: 81-102

63 Wang $\mathrm{X}, \mathrm{Lu} \mathrm{Q}$, Chen $\mathrm{C}$, et al. A consecutive spray printing strategy to construct and integrate diverse supercapacitors on various substrates. ACS Appl Mater Interfaces, 2017, 9: 28612-28619

64 Liu C, Yu Z, Neff D, et al. Graphene-based supercapacitor with an 
ultrahigh energy density. Nano Lett, 2010, 10: 4863-4868

65 Zhong J, Meng J, Yang Z, et al. Shape memory fiber supercapacitors. Nano Energy, 2015, 17: 330-338

$66 \mathrm{Hu}$ X, Fan L, Qin G, et al. Flexible and low temperature resistant double network alkaline gel polymer electrolyte with dual-role $\mathrm{KOH}$ for supercapacitor. J Power Sources, 2019, 414: 201-209

67 Fan E, Li L, Wang Z, et al. Sustainable recycling technology for Li-ion batteries and beyond: Challenges and future prospects. Chem Rev, 2020, 120: 7020-7063

68 Wang Y, Song Y, Xia Y. Electrochemical capacitors: Mechanism, materials, systems, characterization and applications. Chem Soc Rev, 2016, 45: 5925-5950

69 Shao Y, El-Kady MF, Sun J, et al. Design and mechanisms of asymmetric supercapacitors. Chem Rev, 2018, 118: 9233-9280

Acknowledgements This work was supported by the Natural Science Foundation of Tianjin (18JCJQJC46300 and 19JCZDJC31900), the National Natural Science Foundation of China (51822205 and 21875121), the Ministry of Science and Technology of China (2019YFA0705600 and 2017YFA0206701), the Ministry of Education of China (B12015), and the "Frontiers Science Center for New Organic Matter", Nankai University (63181206). The authors thank Professor Zhou Z (Nankai University) for supporting Materials Studio calculations.

Author contributions Wang R performed the experiments and wrote the original manuscript; Yao $\mathrm{M}$ contributed to the electrochemical measurements and revised the manuscript; Huang $S$ and Tian J contributed to the synthesis of electrolytes; Niu Z proposed the concept, supervised the experiments and revised the manuscript. All authors contributed to the general discussion.

Conflict of interest The authors declare that they have no conflict of interest.

Supplementary information Supporting data are available in the online version of the paper.

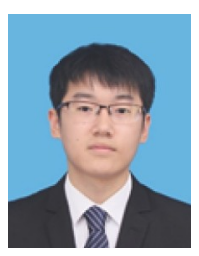

Rui Wang received his BS degree in chemistry from Nankai University in 2018. He then joined the Key Laboratory of Advanced Energy Materials Chemistry at Nankai University under the supervision of Prof. Zhiqiang Niu. His research focuses on the design of smart electrolytes for energy storage devices.

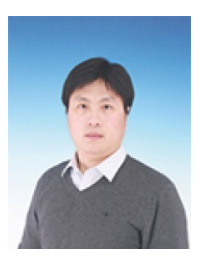

Zhiqiang Niu is a professor at the College of Chemistry, Nankai University. He received his $\mathrm{PhD}$ degree from the Institute of Physics, Chinese Academy of Sciences in 2010. After his postdoctoral research at the School of Materials Science and Engineering, Nanyang Technological University (Singapore), he started his independent research career at Nankai University in 2014. His research interests focus on the unconventional energy storage devices from electrode materials to device configurations.

\section{基于耐低温、保湿多功能凝胶电解质的柔性水系锌 离子电池}

王瑞, 姚敏杰, 黄朔, 田金磊, 牛志强

摘要 水系锌离子电池由于具有安全性高、成本低的特点, 在柔性储 能领域得到广泛关注. 然而, 传统柔性水系锌离子电池处于零下温度 时, 由于其电解质的凝固, 电池容量会发生严重衰减. 此外, 当电池承 受大幅度的形变后, 其外包装易发生破损, 造成电解质中水分的挥发, 最终导致器件失效. 在本文中, 我们开发了一种基于聚丙烯酰胺(PAM) 和甘油(Gly)的耐低温、保湿凝胶电解质. PAM和Gly与水分子之间的 强氢键作用不仅抑制了凝胶电解质在低温时的凝固, 而且限制了电解 质中自由水的挥发. 基于该电解质的柔性水系锌离子电池在 $-40^{\circ} \mathrm{C}$ 时 仍具有优良的电化学性能, 并且无封装的电池在 30 天后仍保持了初始 容量的 $98 \%$. 该工作提供了一种设计水系储能器件用耐低温、保湿凝 胶电解质的新思路. 